\title{
'You should at least ask'. The expectations, hopes and fears of rare disease patients on large-scale data and biomaterial sharing for genomics research
}

\author{
Pauline McCormack ${ }^{\star}, 1$ Anna Kole ${ }^{2}$, Sabina Gainotti ${ }^{3}$, Deborah Mascalzoni ${ }^{4}$, Caron Molster ${ }^{5}$, \\ Hanns Lochmüller ${ }^{6}$ and Simon Woods ${ }^{1}$
}

Within the myriad articles about participants' opinions of genomics research, the views of a distinct group - people with a rare disease (RD) - are unknown. It is important to understand if their opinions differ from the general public by dint of having a rare disease and vulnerabilities inherent in this. Here we document RD patients' attitudes to participation in genomics research, particularly around large-scale, international data and biosample sharing. This work is unique in exploring the views of people with a range of rare disorders from many different countries. The authors work within an international, multidisciplinary consortium, RD-Connect, which has developed an integrated platform connecting databases, registries, biobanks and clinical bioinformatics for RD research. Focus groups were conducted with 52 RD patients from 16 countries. Using a scenario-based approach, participants were encouraged to raise topics relevant to their own experiences, rather than these being determined by the researcher. Issues include wide data sharing, and consent for new uses of historic samples and for children. Focus group members are positively disposed towards research and towards allowing data and biosamples to be shared internationally. Expressions of trust and attitudes to risk are often affected by the nature of the RD which they have experience of, as well as regulatory and cultural practices in their home country. Participants are concerned about data security and misuse. There is an acute recognition of the vulnerability inherent in having a RD and the possibility that open knowledge of this could lead to discrimination.

European Journal of Human Genetics (2016) 24, 1403-1408; doi:10.1038/ejhg.2016.30; published online 6 April 2016

\section{INTRODUCTION}

Widespread use of next-generation sequencing (NGS) is changing the landscape of rare disease research. As technologies become faster and cheaper, the identification of disease-modifying genes, understanding of rare disease mechanisms and investigation into therapeutic approaches have all accelerated. ${ }^{1}$ Patients, data and samples for individual disorders are both scarce and diverse and the relative rarity of resources means collaboration and transnational working are key to research, which aims to improve understanding of $\mathrm{RD}$, advance care and develop therapies. ${ }^{2,3}$

Patient registries and biobanks are recognised as valuable resources for researching the c. $6000 \mathrm{RDs}$, but they have traditionally been organised around single diseases and often replicated in different regions. Unless the required data can be found in one of the few collaborative ventures (EuroBioBank, TREAT-NMD), a researcher either has access to only a small set of local data and samples or undertakes the time-consuming task of requesting samples and/or data from individual repositories. ${ }^{4-7}$

The founding of the International Rare Disease Research Consortium (IRDiRC) in 2011 and the distribution of $€ 144 \mathrm{~m}$ of funding for RD research, via the European's Commission's 7th Framework Programme (FP7), have provided significant impetus for rare disease research and with it, 'a strong rallying call for openness' ${ }^{8}$ Three of the projects funded by FP7 - RD-Connect, NeurOmics and EURenOmics - concentrate on omics approaches to $\mathrm{RD}$ and provide infrastructure to allow new and existing $\mathrm{RD}$ resources to be connected. This work is part of RD-Connect which is linking databases, registries, biobanks and clinical bioinformatics data into a single resource internationally. Practically this means collating details of available $\mathrm{RD}$ research data and biosamples into a searchable online catalogue, ensuring data are interoperable and providing ethical, legal and logistical frameworks that will allow access via a global platform. ${ }^{9,10}$

Collation and distribution of personal genomics data and biosamples raises issues related to patient and public trust. ${ }^{11-13}$ Understanding what those issues are and how they affect patients' and the public's perceptions of an international database is intrinsic to the success of such a platform. ${ }^{14,15}$ To this end, this exploratory study documents the hopes, expectations and concerns of RD patients, as identified by participants themselves, in the changing landscape of NGS and international data sharing.

\section{METHODS}

This article uses data from five focus groups conducted during the EURORDIS Membership Meeting at the European Conference on Rare Diseases 2014 in Berlin and the EURORDIS Summer School for Expert Patients 2014 in Barcelona. EURORDIS is an alliance of rare disease patient organisations with

${ }^{1}$ PEALS Research Centre, Newcastle University, Newcastle upon Tyne, UK; ${ }^{2}$ EURORDIS, Paris, France; ${ }^{3}$ Istituto Superiore di Sanità, Rome, Italy; ${ }^{4}$ Centre for Research Ethics \& Bioethics, Uppsala University, Uppsala, Sweden; ${ }^{5}$ Office of Population Health Genomics, Perth, Western Australia, Australia; ${ }^{6}$ John Walton Muscular Dystrophy Research Centre, Institute of Genetic Medicine, Newcastle University, Newcastle upon Tyne, UK

*Correspondence: Dr P McCormack, PEALS Research Centre, Newcastle University, 4th Floor Claremont Bridge, Newcastle upon Tyne NE1 7RU, UK. Tel: +44 1912083261. E-mail: pauline.mccormack@ncl.ac.uk

Received 19 November 2015; revised 27 January 2016; accepted 1 March 2016; published online 6 April 2016 
almost 700 member organisations across Europe. Sampling for the focus groups was purposive and targeted existing EURORDIS member organisations and most participants have personal or familial experiences of genetic technologies.

Fifty-two people from 16 different countries opted to join the focus groups 32 were women and 20 were men (Table 1). Fifty of the participants were either people with a rare disease or the parent of a child/children with a rare disease and sometimes both. Two people were health professionals working in rare diseases.

This was an exploratory study and therefore did not have a starting hypothesis. Instead, the participants raised issues which they considered of interest, rather than discussing specific topics decided by the researchers. This is a useful technique where so little is known about rare disease patients' views on international data sharing and helps to identify and describe RD patients' ideas and assumptions on this subject. The use of scenarios helps to stimulate the raising of self-identified issues.

Focus groups were chosen for the ability to allow in-depth discussion among people with common interests, whether opinions are heterogeneous or homogeneous. In addition, it is thought that the format puts participants at ease which benefits the raising of sensitive or contentious issues. ${ }^{16,17}$

The focus groups used two scenarios based on real life problems, which were the result of exploratory work in RD-Connect involving project researchers, the Patient Advisory Council (PAC) and the Patient and Ethics Council (RD-PEC). The first scenario was based on the reuse of historical consents and issues around re-consenting patients in order to repurpose historical samples for a new study. The second scenario featured the Care.data scheme overseen by the National Health Service in England, which aimed to compile a national database of medical records from primary care organisations and hospitals, for use in research. The implementation of the Care.data scheme is delayed and highly criticised for failure to communicate adequately with the public (http:// www.bbc.co.uk/news/health-26259101). This example was used to stimulate discussion about what information people seek when considering participation in a large database for health research. The scenarios were designed to allow participants, rather than the researcher, to identify the specific topics for discussion and to talk about their own viewpoints and experiences.

Scenarios are less restrictive than surveys and allow debate or conversations to be opened up between participants where they are given the opportunity to talk about their own behaviours, opinions and beliefs. ${ }^{18,19}$ They allow free participation, especially around sensitive or difficult topics, where focus group members can choose to be explicit about their own experiences or simply to draw on those, while at the same time keeping comments focussed on the scenario in hand. ${ }^{20}$

Focus groups were moderated, digitally recorded and lasted 60-90 minutes. Analysis was inductive but within a framework of previous exploratory work carried out in previous workshops with clinicians, scientists, bioethicists, patient advocates and industry representatives involved with RD-Connect, NeurOmics and EURenOmics.

\section{Ethical approval}

All procedures performed in studies involving human participants were in accordance with the ethical standards of the institutional and/or national research committee and with the 1964 Helsinki declaration and its later amendments or comparable ethical standards.

Table 1 Focus group participants by country of residence

\begin{tabular}{llcl}
\hline France & 9 & Denmark & 2 \\
UK & 9 & Portugal & 2 \\
Germany & 8 & Slovakia & 2 \\
Italy & 4 & Iceland & 2 \\
Serbia & 3 & Belgium & 1 \\
Netherlands & 3 & Czech Rep & 1 \\
Greece & 2 & Ireland & 1 \\
USA & 2 & Romania & 1 \\
\hline
\end{tabular}

\section{RESULTS}

\section{Summary}

The members of the focus groups were positively disposed towards participating in research and allowing their own data and biosamples, as well as those of any affected children, to be shared internationally. All the participants understood the incentive for $\mathrm{RD}$ in sharing data and samples; in fact, there were several pleas for research systems to be standardised across the EU in order to make data sharing easier.

Before participants would be willing to participate in a centralised database, they would seek information about governance and management, especially around who has access to data in the platform. In addition, participants were interested in how patient advocacy organisations would be involved in the ongoing supervision of a global platform for RD.

Individuals' levels of trust and attitudes to risk differed, and while a large majority of the participants were concerned about threats to privacy and autonomy, the scale and scope of these concerns varied between individuals. Individuals' approaches were often bounded culturally and this included: politically and socially mediated practices and norms in the country in which they lived; the characteristics of the rare disease that they and/or their child had; and individual experiences with research.

All these influences made participants reflect on data sharing and linking, including the benefits and risks, in slightly different contexts. They quoted recent examples of perceived misuse of technology or leaking of data as background to ongoing concerns about the privacy of their data in any system, such as revelations by former CIA employee Edward Snowden that USA intelligence services routinely conducted illegal surveillance of individuals' data (http://www. theguardian.com/us-news/the-nsa-files).

The participants understand that futures can be uncertain and the nature of that uncertainty cannot be predicted, and in light of this, they look for safeguards and stability within research systems, which will go some way towards ameliorating risks. The discussion here highlights four themes that emerged from the data and illustrate factors that are important to RD patients and families when they are considering participation in research.

\section{Consent for historical samples and data}

Using the scenario example of a clinician-researcher assessing whether or not she should re-contact and re-consent participants to allow the re-use of historic samples and data, most participants felt very strongly that participants should be re-contacted if the specific usage was not covered in the original consent. The introduction of certain conditions, such as the anonymisation of samples or the disorder being ultra-rare, did not affect their viewpoint on this.

A variety of reasons were invoked, including genetic exceptionalism, which is the notion that genetic data has particular importance and should be treated differently to other health data ${ }^{21}$ and the possibility that the new use might not fit with the participant's values, for example, using embryonic stem cells. This is the notion of 'you should at least ask', and it can be discerned that the decision to participate in a new study should be the patient's/parent's alone and that this decision should be reached without the invocation of persuasive elements, such as the study being for an ultra-rare condition.

To most participants, the possibility of the researcher applying to a research ethics committee to proceed without re-consenting individuals was seen as a threat to both individual and group autonomy and to trust. In addition, there is the notion here of group solidarity where the participant feels beholden to the group to behave in a manner morally acceptable to other parents. Other participants evoked equality 
of treatment across all samples in the study, "The, um, samples that you're taking at the moment, presumably, she would have to go through having everybody sign an informed consent form ... She would have to go through that process with the new ones. Right? ... So you can't have a two tier situation with one group giving open consent and the other group being assumed not to have consent".

Several participants see informed consent as a contract where both sides promise to honour the agreement, and that to proceed with a study without re-consenting participants is to modify the terms of this agreement without the knowledge of the other party.

Although all the participants recognised the benefit of making the most of rare samples that can be difficult to re-collect, only a minority thought that samples and/or data should be used without re-consent. For these participants, the use of anonymisation and ethical review was sufficient. Justification included that there was no or little risk, that wastage of precious samples and data should be avoided and the resources used for re-consent represented an opportunity cost for some other aspect of the research. In addition, the fact that there are temporal aspects to rare diseases which are progressive and/or lifelimiting, where the move towards a cure is imperative and is a critical factor for families, is seen by a minority of participants to be a driver to making use of as many samples and data as possible.

'I'm probably gonna have complications of this disease exactly the way my grandmother, my great-grandmother did and my mother will, but [my daughter] is two and a half, I hope that in the next fifteen years something comes up before it starts like big time... so the question is, with so little time, personally, I'd say, let's use whatever is there'

\section{Consent-re-consent and children}

There was debate within the focus groups about whether children or their families should be contacted to seek re-consent when a child comes of age. There were broadly two sets of views, the first was that the original consent is legitimate and should stand without re-contact, and the second that it was unacceptable to reuse a sample for someone who was now an adult and had not consented for themselves. Arguments put forward for the first view included opposition to project resources being spent on re-contact and re-consent where there was no requirement to do so as well as the consideration that re-contact could be upsetting for a family if the child had died. Participants in favour of re-consent thought that re-contact was desirable in any circumstances - that the child-now-adult's autonomy should be respected by giving them an opportunity to make their own decision about participation.

Counter to the previous argument, this group thought that ongoing communication with the child-now-adult and the family would be beneficial and would show that research into the condition was ongoing and the child's sample still had utility and that re-contact had the role of respecting the family's autonomy.

'I think it could be an opportunity to let them know that we are still fighting to find drugs or something better for new patients who have the same disease as their daughter or son and I think ... let people know that we are still looking for a solution and we are also looking for fundraising, for everything like this and it's an opportunity'.

\section{Data sharing and access to data}

Use of a scenario based on the attempted implementation of the Care. data scheme in England prompted discussion around the conditions under which data sharing should take place. Most of the focus group attendees would not give permission for their records to be used under the broad and indeterminate circumstances illustrated by the scenario and would be unhappy with an opt-out system. In order to join or give consent for their data to be included in a 'broad use' database, they would seek specific information on curatorship, governance and details about access and security. Participants were in favour of systems run by public or not-for-profit organisations, such as universities, hospitals and clinics.

Most participants would like to limit access to their records to people working in health care and health research. All participants were against the data being accessed by private companies. Even when prompted that pharmaceutical companies need access to patient data and records for drug development and clinical trials, participants were of the view that this should only be allowed in partnership and via not-for-profit health organisations.

'I'm very worried about independence, about studies in general, I'm very worried about pharmaceutical companies coming in... so I wouldn't like this to become, I'm selling you a list of people with this, I don't know, I just, I feel uncomfortable. It would take too long to explain all my point of views but that makes me feel very, very uncomfortable, first and because I don't feel protected'.

Participants were also concerned about the stability of private companies, and this was illustrated by an Icelandic participant who had recently been approached by deCODE to provide a sample for genomic analysis to add to their database. She prefaced her story by stating that deCODE's founder was severely affected by the Icelandic economic crash of 2008. In fact, deCODE filed for bankruptcy at this time and were subsequently acquired by the American company Amgen and, in turn, by the Chinese company WuXi Pharma Tech in 2015. There is a tension between what participants see as the private nature of people's health records and DNA and the international, market-driven approach of a company.

\section{Management, governance and security}

Participants' high levels of criticism about what they saw as vague assurances and a lack of detail given in the Care.data example emphasises the need for detailed, relevant information and also that different people require information about different areas of governance and management. Some focus group participants were more interested in knowing that researcher's activity on the system was tracked, whereas others would seek technical detail on types of security used for the whole system. One participant likened the situation to a bank, where you deposit your money on the grounds that there are agreed levels of protection for your funds.

'I'm just trying to say there is this framework, you know we say that there is a governance system in place which will protect the patient and we can look at them like we do the financial institutions and we're quite happy with how they exist, well they're quite well developed. There's a frameworks around this and we want some assurance'.

Participants seek protection from: their data being used for a purpose for which they did not consent; the possibility of discrimination through their data being made available to an employer or government department; and from other uncertainties. There was a particular concern about the possibility of insurance companies 
gaining access to data and using this to make decisions with regard to the provision of health or other types of insurance.

There was an acute recognition, among those taking part in the focus groups, of the vulnerability inherent in being identified as having a rare disease and possibility that open knowledge of this could lead to discrimination in a number of quarters, such as employment or the provision of financial services.

\section{Inclusion of patient organisations in governance}

One of the means of meeting participants' expectations of protection is to ensure that patient organisations are represented in ongoing governance of a global database. Focus group attendees were of the view that the experiential knowledge of patient groups could contribute to good governance of a global platform.

'Well I am just coming back on this capacity development for patient organisation-I think it's a very key, not just with this project but with many other projects-if we want to control, to have a good governance of this project, we need to have citizen and patient on board and we need to raise their capacity'.

Some participant's ideas of collaboration went further than this as they thought that patient organisations had a duty to encourage clinicians and researchers to collaborate in populating a global platform for research.

'I think this is our task, to put them together and now I think, there are changes in the mind of the doctors ... there is a lot of work to do, but we only can do it together with you, so it's our task to tell them that they are open-minded, and to convince other doctors to be open-minded, and I think there is really the change now'.

\section{Risk/benefit}

There is a strong notion among the focus groups' discussion of personal choice in that participants take decisions which they deem correct for themselves or their child and which they feel comfortable with. They recognise that others, even in the same circumstances, might feel and therefore act differently. One participant gave the analogy that some people willingly use credit cards online while others avoid this and that participants undertake their own risk/benefit audit in each circumstance.

Participants who value the benefits of data and sample sharing as outweighing any potential risks often do so on an individual basis and are not prepared to take a position on behalf of the patient organisation they represent but rather highlight the importance of each patient making such decisions for themselves.

Risk versus benefit decisions can also be informed by the characteristics of the disease as illuminated in a lively debate during the focus groups between two mothers of children with $\mathrm{RD}$. The mother of a child with a progressive, life-limiting disorder was prepared to be relatively permissive about giving consent and sharing data on the grounds that, 'our biggest vulnerability is the fact that we don't have a cure. We don't have... We don't have time, that's the way I see it'.

In direct contrast, a mother of a child with an inherited intellectual disability was concerned about discrimination and used the example of past cultural norms of societal exclusion of people with intellectual disability as a reason to proceed with caution in sharing data which identifies such difference '...but what about, inherited intellectual disabilities, which years ago, like people were institutionalised...so it's a just different way of thinking for intellectual disability'.

Participants cited many other risks, such as being re-identified by a researcher compiling records from several different sources and unattributed paternity. Many of them though talked about multiple aspects of daily life - employment, insurance (including health insurance), financial services and health-care provision - where they feared being discriminated against if access to data was not strictly controlled.

\section{DISCUSSION}

Opinions on consent in these focus groups indicate that participants are keen to retain autonomy, for themselves and for the group they might represent, by being given the opportunity to consent or re-consent when research purposes change. Some see consent as a contract which should be honoured by both sides. Consent for medical research is a type of contract and has legal status and conditions, which are legally enforceable. ${ }^{22}$ It would appear though that members of the focus groups are not thinking solely of a functional, legal role for consent and have moved beyond the gift relationship ${ }^{23}$ to seeing consent as a social agreement where they reject that decisions should be taken by a researcher or ethics board. ${ }^{24,25}$

This is especially important given current debates around broad consent versus dynamic consent. ${ }^{26}$ Many rare disease patients and their families have prolonged contact with research teams and there are strong notions in the findings of a duty to participate in order to aid research progress. It seems that participants expect clinicians and researchers to have concordant duties, including consulting with the patient/family if there is a possibility that new research moves beyond the bounds of the original informed consent. According to Gottweis et $a l,{ }^{27}$ 'people need to feel that they are part of something larger and that their donation feeds into a mutual, respectful relationship'.

This mutuality extends to the idea of renewing contact when a child is potentially coming of age. Although there is no current legal requirement for children to be re-consented on reaching adulthood, the general consensus in the ethics literature ${ }^{28}$ is that children should be re-consented when they reach the age of majority or provided with information and the opportunity to opt out. In a recent study among young adult cancer survivors, whose tissue and data was lodged in a biobank when they were children, $70 \%$ were unaware of their participation yet $100 \%$ agreed their tissue could continue to be used, illustrating the importance of re-contact for their individual autonomy as adults and for mutuality of decision-making. ${ }^{29}$

Focus group participants saw re-contact as crucial in all circumstances and re-contact was viewed as a positive interaction. These ongoing, long-term interactions can build trusting relationships between researchers, clinicians and patients that can ultimately have a positive impact on participation. ${ }^{14,30}$

Concerns about data sharing and access and around private companies is in common with other studies. ${ }^{30-33}$ In many cases, participants were concerned about the risk of stigma and discrimination against themselves or their children that they perceive could have a negative impact on access to health care and other opportunities in life. These are Goffman's discredited citizens, not fully accepted by society, ${ }^{34}$ where 'families have the capacity to construct a 'protective capsule' for their stigmatised child's identify formation, through the control of information to the child and to the outside community'. ${ }^{35}$

The pernicious effects of discrimination are real and recent experiences for many people with rare diseases and their 
families, ${ }^{36,37}$ and while genetic discrimination is banned in most of the countries that our participants are from, it is not always present in legislation or treated in the same manner throughout the EU, United States of America and Canada. ${ }^{38}$

It is therefore important for rare disease patients and families to mitigate, as far as possible, against uncertainties that might lead to their data 'getting into the wrong hands'. Participants have high levels of trust in public institutions ${ }^{39}$ and expect such organisations to protect against the possibility of participation in a global database leading to disadvantage, especially given concerns about possible, unknown changes in political landscape, regulation or legislation.

Given that most of the participants in this study were involved in executive or management activities in a rare disease organisation and many were operating proficiently with English as a second language, they are not likely to be from a wide variety of social backgrounds. It was vital for this research that participants understood how RD research infrastructures operate and it was therefore necessary to focus on these 'expert patients'. It is of course possible that RD patients and families from different socio-economic backgrounds, or with a worldview that does not include a role as a RD advocate, would have different opinions from the focus group participants.

That said, they represent a significant number of rare disease representatives for a qualitative study and are in a position to provide important insight into issues around genomic technologies and international data sharing that are likely to affect all research in the future and not just the field of rare disease.

\section{CONCLUSION}

In this analysis, we have established that rare disease patients and parents see their dealings with researchers as motivated by common interests, and participants are keen to make their samples and data available to researchers as long as this action is treated with respect and reciprocity.

As their data and samples are shared and used by researchers around the world, participants could perceive that the locus of influence has shifted and their control over information about their and their family's health is diminished. Hence, the strongly expressed desire to protect their and their children's autonomy and to protect against lapses in privacy and the potential for subsequent discrimination.

One of the means of doing this is to ensure that patient organisations are represented in ongoing governance of a global platform such as $\mathrm{RD}$-Connect as part of ensuring that participants feel they have an equivalent level of protection and control in these global interactions as they do in their local relationships with researchers.

\section{CONFLICT OF INTEREST}

The authors declare no conflict of interest.

\section{ACKNOWLEDGEMENTS}

We thank the RD Patient and Ethics Council and RD-Connect Patient Advisory Council for assistance with research design (Jean Jacques Cassiman, Tracy Dudding, Muriel Gevrey, Emma Heslop, Joseph Irwin, Julian Isla, Sigurður Jóhannesson, Lydia Lemonnier, Chantal Loirat, Dorthe Lykke, Milan Macek, Kay Parkinson, Odile Perrousseaux, Marita Pohlschmidt, Daniel Renault, Peter Reussner, Francoise Rouault, Balthasar Schaap, Inge Schwersenz, Chris Sotirelis, Volker Straub, Oliver Timmis, Marieke van Meel, Elizabeth Vroom) and Cathy Turner for her invaluable contribution. The study was supported by the Medical Research Council UK (reference G1002274, grant ID 98482) and by the European Union Seventh Framework Programme (FP7/2007-2013) under grant agreement nos. 305444 (RD-Connect) and 305121 (Neuromics).

1 Boycott KM, Vanstone MR, Bulman DE, MacKenzie AE: Rare-disease genetics in the era of next-generation sequencing: discovery to translation. Nat Rev Genet 2013; 14: 681-691.

2 Lochmüller $\mathrm{H}$, Aymé S, Pampinella $\mathrm{F}$ et al: The role of biobanking in rare diseases: European consensus expert group report. Biopreserv Biobank 2009; 7: 155-156.

3 McCormack P, Woods S, Aartsma-Rus A et al: Guidance in social and ethical issues related to clinical, diagnostic care and novel therapies for hereditary neuromuscular rare diseases: "translating" the translational. PLoS Curr 2013; e-pub ahead of print 10 January 2013; doi:10.1371/currents.md.f90b49429fa814bd26c5b22b13d773ec.

4 Forrest CB, Bartek RJ, Rubinstein Y, Groft SC: The case for a global rare-diseases registry. Lancet 2011; 377: 1057-1059.

5 Bladen CL, Rafferty K, Straub V et al: The TREAT-NMD Duchenne Muscular Dystrophy Registries: conception, design, and utilization by industry and academia. Hum Mutat 2013; 34: 1449-1457.

6 Monaco L, Crimi M, Wang CM: The challenge for a European network of biobanks for rare diseases taken up by RD-connect. Pathobiology 2014; 81: 231-236.

7 Peixoto S: Orphanet: Rare Disease Registries in Europe. Paris: Orphanet, 2015.

8 Lochmüller H, Thompson R: Rare diseases need global solutions: new international initiatives in rare disease omics research. BJSM News 2013; 49: 2-3.

9 Mascalzoni D, Dove ES, Rubinstein $Y$ et al: International Charter of principles for sharing bio-specimens and data. Eur J Hum Genet 2015; 23: 721-728.

10 Knoppers BM: Framework for responsible sharing of genomic and health-related data. HUGO J 2014; 8: 3.

11 Darquy S, Moutel G, Lapointe A-S et al: Patient/family views on data sharing in rare diseases: study in the European LeukoTreat project. Eur J Hum Genet 2015; 24: 338-343.

12 Genetic_Alliance_UK: Genome Sequencing: What do Patients Think?. London, UK: Genetic Alliance UK, 2015.

13 Tabor HK, Stock J, Brazg T et al: Informed consent for whole genome sequencing: a qualitative analysis of participant expectations and perceptions of risks, benefits, and harms. Am J Med Genet A 2012; 158A: 1310-1319.

14 Johnsson L, Helgesson G, Hansson MG, Eriksson S: Adequate trust avails, mistaken trust matters: on the moral responsibility of doctors as proxies for patients' trust in biobank research. Bioethics 2012; 27: 485-492.

15 McCormack P, Kole A: Setting up strategies: patient inclusion in biobank and genomics research in Europe. Orphanet J Rare Dis 2014; 9: P2.

16 Khan ME, Anker M, Patel BC, Barge S, Sadhwani H, Kohle R: The use of focus groups in social and behavioural research: some methodological issues. World Health Stat $Q$ 1991; 44: 145-149.

17 Clavering EK, McLaughlin J: Crossing multidisciplinary divides: exploring professiona hierarchies and boundaries in focus groups. Qual Health Res 2007; 17: 400-410.

18 Hazel N: Elicitation techniques with young people. Soc Res Updat 1995; 12.

19 Finch J: The vignette technique in survey research. Sociology 1987; 21: 105-114.

20 Barter C, Renold E: 'I wanna tell you a story': exploring the application of vignettes in qualitative research with children and young people. Int J Soc Res Methodol 2000; 3 307-323

21 Rothstein MA: Genetic exceptionalism \& legislative pragmatism. Hastings Cent Rep 2005; 35: 27-33.

22 Leonhard C: The unbearable lightness of consent in Contract Law. Case W Res $L$ Rev 2012; 63: 57.

23 Oakley A, Ashton J: The Gift Relationship: From Human Blood to Social Policy. London, UK: London School of Economics and Political Science, (LSE), 1997.

24 Hayden EC: A broken contract. Nature 2012; 486: 312-314.

25 O'Doherty $\mathrm{KC}$, Burgess MM, Edwards $\mathrm{K}$ et al: From consent to institutions: designing adaptive governance for genomic biobanks. Soc Sci Med 2011; 73 367-374

26 Garrison NA, Sathe NA, Antommaria AHM et al: A systematic literature review of individuals' perspectives on broad consent and data sharing in the United States. Genet Med 2015; e-pub ahead of print 19 November 2015; doi:10.1038/gim.2015.138

27 Gottweis H, Gaskell G, Starkbaum J: COMMENT connecting the public with biobank research: reciprocity matters. Nat Rev Genet 2011; 12: 738-739.

28 Hens K, Levesque E, Dierickx K: Children and biobanks: a review of the ethical and legal discussion. Hum Genet 2011; 130: 403-413.

29 Rush A, Battisti R, Barton B, Catchpoole D: Opinions of young adults on re-consenting for biobanking. J Pediatrics 2015; 167: 925-930.

30 Levy D, Splansky GL, Strand NK et al: Consent for genetic research in the Framingham Heart Study. Am J Med Genet A 2010; 152A: 1250-1256.

31 Laur A: Fear of e-Health records implementation? Med Leg J 2014. 83. 34-39.

32 Lemke AA, Wolf WA, Hebert-Beirne J, Smith ME: Public and biobank participant attitudes toward genetic research participation and data sharing. Public Health Genomics 2010; 13: 368-377.

33 McGuire AL, Hamilton JA, Lunstroth R, McCullough LB, Goldman A: DNA data sharing: research participants' perspectives. Genet Med 2008; 10: 46-53.

34 Goffman E: Stigma. Englewood Cliffs, NJ, USA: Spectrum, 1963.

35 Carnevale FA: Revisiting Goffman's stigma: the social experience of families with children requiring mechanical ventilation at home. J Child Health Care 2007; 11: 7-18. 
36 Lapham EV, Kozma C, Weiss JO: Genetic discrimination: perspectives of consumers. Science 1996; 274: 621-624.

37 Geelen E, Horstman K, Marcelis CL, Doevendans PA, Van Hoyweghen I: Unravelling fears of genetic discrimination: an exploratory study of Dutch HCM families in an era of genetic non-discrimination acts. Eur J Hum Genet 2012; 20: 1018-1023.
38 Office_of_the_Privacy_Commissioner_of_Canada: Genetic Information, the Life and Health Insurance Industry and the Protection of Personal Information: Framing the Debate. Gatineau, QC, Canada: Office of the Privacy Commissioner of Canada, 2012. 39 Gottweis H, Chen H, Starkbaum J: Biobanks and the phantom public. Hum Genet 2011; 130: 433-440. 